\title{
ON THE $L_{p}$-DECAY AND LOCAL ENERGY DECAY OF SOLUTIONS TO NONLINEAR KLEIN-GORDON EQUATIONS
}

\author{
PHILIP BRENNER \\ School of Mathematical Sciences, Chalmers and Göteborg University \\ SE 41296 Göteborg, Sweden \\ E-mail: philip@math.chalmers.se
}

The Klein-Gordon equation is the equation for relativistic wave-propagation

$$
\begin{gathered}
\partial_{t}^{2} u_{o}-\Delta u_{o}+m^{2} u_{o}=0, \quad x \in \mathbf{R}^{n}, t \geq 0 \\
\left.u_{o}\right|_{0}=\varphi,\left.\partial_{t} u_{o}\right|_{0}=\psi, \quad x \in \mathbf{R}^{n},
\end{gathered}
$$

where $m>0, \Delta=\sum_{j=1}^{n} \partial_{x_{j}}^{2}(n \geq 3)$. The nonlinear counterpart, extensively studied since early 1960 's, is

(NLKG)

$$
\begin{gathered}
\partial_{t}^{2} u-\Delta u+m^{2} u+f(u)=0, \quad x \in \mathbf{R}^{n}, t \geq 0 \\
\left.u\right|_{0}=\varphi,\left.\partial_{t} u\right|_{0}=\psi, \quad x \in \mathbf{R}^{n},
\end{gathered}
$$

where $f(u)$ is a nonlinear function, $f(u) \cong|u|^{\rho-1} u$; modified at 0 if necessary, to be smooth enough, and with

$$
1+\frac{4}{n}<\rho<\frac{n+2}{n-2}=\rho^{*}
$$

The conditions on $f$ will be made precise below.

Our paper will describe the behavior of local energy and of $L_{p}$-norms of solutions to the KG and NLKG for large values of time. We will assume that $F(u)=\int_{0}^{u} f(v) d v \geq 0$. The energy

$$
E(t)=\frac{1}{2} \int\left(\left|\partial_{x} u\right|^{2}+\left|\partial_{t} u\right|^{2}+m^{2}|u|^{2}\right) d x+\int F(u) d x
$$

is a conserved quantity, $E(t)=E(0)$. Let $X_{e}=H_{2}^{1} \times L_{2}$ with norm $\|\cdot\|_{e}$ defined by

$$
\|u(t)\|_{e}^{2}=\|u(t)\|_{H^{1}}^{2}+\left\|\partial_{t} u(t)\right\|_{L_{2}}^{2} .
$$

Our assumptions on $\rho$ and $f$ imply that

$$
E(t) \leq C\|u(t)\|_{e}^{2}
$$

2000 Mathematics Subject Classification: Primary 46E35; Secondary 35L05.

The paper is in final form and no version of it will be published elsewhere. 
Correspondingly, for $\Omega \subset \mathbf{R}^{n}$ we define the local energy by

$$
E_{\Omega}(t)=\frac{1}{2} \int_{\Omega}\left(\left|\partial_{x} u\right|^{2}+\left|\partial_{t} u\right|^{2}+m^{2}|u|^{2}+2 F(u)\right) d x
$$

and $X_{e}(\Omega)$ is defined by replacing the global spaces and norms in the definition of $X_{e}$ by the corresponding local spaces and norms. The local energy as defined is no longer a conserved quantity (to get conservation of energy, one has to work with the whole surface of the light cone).

Global estimates in space-time (Strichartz estimate) for the KG (Strichartz [19], Segal $[15], \ldots)$ : If the data $\varphi, \psi$ belong to $X_{e}$, then

$$
\left\|u_{o}\right\|_{L_{p}\left(L_{p}^{1 / 2}\right)} \leq C\left(\|\varphi\|_{H^{1}}+\|\psi\|_{L_{2}}\right) \leq C\left\|u_{o}(0)\right\|_{e}
$$

where $p \geq 2, \delta_{p}=\frac{1}{2}-\frac{1}{p}=\frac{1}{n+1}$. More general, but also much more complex, estimates that bound $u_{0}$ in $L_{q}\left(\mathbf{R}, H_{p}^{s}\left(\mathbf{R}^{n}\right)\right)$ are available (Strichartz [19], Marshall-Strauss-Wainger [11], Brenner [2]; a good exposition is given by Ginibre and Velo [6]). One such example we will use is that if the data belong to $X_{e}$ then

$$
\begin{gathered}
u_{o} \in L_{2}\left(\mathbf{R}, H_{p}^{\gamma}\left(\mathbf{R}^{n}\right)\right) \\
\text { where } \delta=\frac{1}{2}-\frac{1}{p} \in\left(\frac{1}{n}, \frac{1}{n-1}\right) \text { and } \gamma=\frac{1}{2}-\frac{1}{n-1} .
\end{gathered}
$$

Space-time integrals of solutions of NLKG. Let $u_{o}$ be a solution of KG with the same data at $t=0$ as $u$, the solution of NLKG. Assume that the data has finite energy (i.e. $u(0), \partial_{t} u(0)$ belong to $\left.X_{e}\right)$. Then one example of a Strichartz-type estimate for the NLKG due to the author [4] is that if

$$
\begin{gathered}
\delta=\frac{1}{2}-\frac{1}{p}, 0 \leq \sigma \leq s \leq 1, \theta \in(0,1], \\
\frac{s-\sigma}{1-\sigma}<\rho-1,(n+1+\theta) \delta \leq 1+s-\sigma, \\
(n-1-\theta) \delta<1<(n-1+\theta) \delta
\end{gathered}
$$

then

$$
\text { if } u_{o} \in L_{q}\left(\mathbf{R}, H_{p}^{\sigma}\left(\mathbf{R}^{n}\right)\right) \text { then } u \in L_{q}\left(\mathbf{R}, H_{p}^{\sigma}\left(\mathbf{R}^{n}\right)\right) \text {. }
$$

In view of our previous example, if the data belong to $X_{e}$, then the conditions above are satisfied for $0 \leq \sigma \leq \gamma$ and $\frac{1}{n}<\delta<\frac{1}{n-1}$, and hence

$$
u \in L_{2}\left(\mathbf{R}, H_{p}^{\gamma}\left(\mathbf{R}^{n}\right)\right) \text {. }
$$

The following is a result on (local) energy decay: Let $\Omega_{t}=\{\epsilon(t) t \leq|x| \leq(1-\epsilon(t)) t\}$, where $0<\epsilon(t)<1, \epsilon(t) \rightarrow 0$, as $t \rightarrow \infty$. Let $Y_{t}=H_{2}^{1}\left(\mathbf{R}^{n} \backslash \Omega_{t}\right)$. Then

Energy Decay Theorem (Strichartz [20]). Let $u_{o}$ be a finite energy solution of the Klein-Gordon equation. Then

$$
\left\|u_{o}(t)\right\|_{Y_{t}} \rightarrow 0 \text { as } t \rightarrow \infty \text {. }
$$

Earlier Morawetz [12] proved a result about energy decay on compact subsets $\Omega$ of $\mathbf{R}^{n}$ : 
Local Energy Decay Theorem (Morawetz [12]). Let $n=3$ and assume that $u$ is a solution of the NLKG, which is locally a classical solution. Then

$$
E_{\Omega}(t) \in L_{1} \text { and } E_{\Omega}(t) \rightarrow 0 \text { as } t \rightarrow \infty \text {. }
$$

In particular,

$$
\|u(t)\|_{L_{2}(\Omega)} \in L_{2} \text { and tends to } 0 \text { as } t \rightarrow \infty \text {. }
$$

We will study the behavior of $u(t)$, a solution of NLKG with finite energy data. Let $\Omega$ be a compact subset of $\mathbf{R}^{n}$, and let $|\Omega|$ denote the volume of $\Omega$ in $\mathbf{R}^{n}$. Then

$$
\|u(t)\|_{L_{2}(\Omega)} \leq|\Omega|^{\delta}\|u(t)\|_{L_{p}(\Omega)}
$$

where as before, $\delta=\frac{1}{2}-\frac{1}{p}$. If $\frac{1}{n}<\delta<\frac{1}{n-1}$ then as mentioned above $\|u(t)\|_{L_{p}} \in L_{2}$, which proves that

$$
\|u(t)\|_{L_{2}(\Omega)} \in L_{2} .
$$

In addition, we may use the existence of solutions of the Klein-Gordon equation approximating $u$ in the following sense

Scattering Theorem (Brenner [2], [3], [4]). There exists an everywhere defined scattering operator on $X_{e}$ for the $N L K G$.

In particular there is a solution $u_{+}$of the Klein-Gordon equation with finite energy such that

$$
\left\|u(t)-u_{+}(t)\right\|_{e} \rightarrow 0 \text { as } t \rightarrow \infty .
$$

We conclude, using that $u_{+}(t)$ is uniformly continuous in $H_{2}^{1}$, that $u$ is uniformly continuous in $L_{2}(\Omega)$. The integrability then implies that

$$
\|u(t)\|_{L_{2}(\Omega)} \rightarrow 0 \text { as } t \rightarrow \infty .
$$

Notice that this result, as well as the extension of the Energy Decay Theorem to the solution of the NLKG, follows from that theorem and the Scattering Theorem above. We have

$$
\|u(t)\|_{Y_{t}} \rightarrow 0 \text { as } t \rightarrow \infty
$$

where again $Y_{t}=H_{2}^{1}\left(\mathbf{R}^{n} \backslash \Omega_{t}\right)$, with $\Omega_{t}=\{\epsilon(t) t \leq|x| \leq(1-\epsilon(t)) t\}$, where $0<\epsilon(t)<1$, $\epsilon(t) \rightarrow 0$ as $t \rightarrow \infty$.

There has been a number of results on pointwise decay in $L_{p}\left(\mathbf{R}^{n}\right)$ of solutions of the NLKG over the years by e.g. Strauss [17], Morawetz and Strauss [13], Pecher [14], Brenner [1], [3], [4], ..

Pointwise $L_{p}$ Decay TheORem (Brenner [4]). Let $u$ be a solution of NLKG with sufficiently nice data (data which have sufficiently many derivatives in $L_{1}$, say). Then if $\delta=\frac{1}{2}-\frac{1}{p}<\min \left(\frac{1}{n-1}, \frac{\rho-1}{4}\right), p \geq 2$, then

$$
\|u(t)\|_{H_{p}^{1}} \leq C(1+t)^{-n \delta} .
$$

The work of Grillakis ([8], [9]) on classical solutions for the nonlinear wave equation for critical exponents $\rho=\rho^{*}$ proves that we in this case may use $\delta=\frac{1}{2}$ for $n=3$ (and $p=\infty)$. This result can be extended to the NLKG. The choice $\delta=\frac{1}{n-1}$ probably also 
holds for $n \leq 6$, even in the critical case $\rho=\rho^{*}$. Using the Pointwise $L_{p}$ Decay Theorem and our previous estimate of $u$ in $L_{p}(\Omega)$, we get

Local Pointwise $L_{p}$ Decay Theorem. Let $n \geq 3$, and let $\delta<\frac{1}{n-1}$. Then

$$
\|u(t)\|_{H_{2}^{1}(\Omega)} \leq C\left(\frac{|\Omega|^{1 / n}}{1+t}\right)^{n \delta}
$$

for sufficiently "nice" data.

Comment. For $n=3$, we may allow $\delta=\frac{1}{2}$, as mentioned above. We hope to come back to this and related questions at a later occasion.

EXAmple. Let $\Omega=\Omega(t)$ with $|\Omega(t)| \leq t^{\alpha n}, 0 \leq \alpha<1$. Then

$$
\|u(t)\|_{H_{2}^{1}(\Omega(t))} \leq C t^{-(1-\alpha) n \delta} .
$$

Notice that only the size, not the actual position of $\Omega$ is involved.

The largest $\delta$-value for which we get decay results for solutions of the NLKG is determined by the singularities of the map

$$
L_{p} \ni v \rightarrow E(t) v \in L_{p}, 1 / p+1 / p^{*}=1,
$$

where the solution of the Klein-Gordon equation is given by $u(t)=F(t) \phi+E(t) \psi$, with $\phi=u(0), \psi=\partial_{t} u(t)$. Much indicates that $\delta \leq \frac{1}{n-1}$, including results on classical solutions for critical case exponents, $\rho=\rho^{*}$. Notice also that the maximal rate of decay for the solutions of the Klein-Gordon equation seems to be $\mathcal{O}\left(t^{-n / 2}\right)$ in $L_{p}(\Omega), \Omega \subset \subset \mathbf{R}^{n}, p \geq 2$. More should not be expected for sets $\Omega \subset\{|x| \leq t\}$, in contrast to the rapid decay of solutions of the wave equation in sets away from the light cone.

We will next give a lower bound for the decay in the context of global $L_{p}$-norms.

Theorem. Let $u \in X_{e}$ be a solution of the NLKG, $n \geq 3$ and with $f$ satisfying conditions (i) through (iii) from Appendix 2. Assume that $u \in L_{q}^{\operatorname{loc}}\left(\mathbf{R}, L_{p}^{\operatorname{loc}}\left(\mathbf{R}^{n}\right)\right)$, with $q, p \geq 2$, and $\delta=\frac{1}{2}-\frac{1}{p}$. Let

$$
X_{t}=L_{p}(\{|x| \leq t\}) \supseteq X=L_{p}\left(\mathbf{R}^{n}\right) .
$$

Then there is a constant $c>0$ such that

$$
\left(\frac{1}{T} \int_{t}^{t+T}\|u(\tau)\|_{X_{\tau}}^{q} d \tau\right)^{1 / q} \geq c t^{-n \delta},
$$

for $t \geq 1$, and $t \geq T>0$.

Comment. We may replace $X_{t}$ in our Theorem by $Y_{t}=L_{p}(\{\epsilon(t) t \leq|x| \leq(1-\epsilon(t)) t\})$ where $\epsilon(t)$ denotes any positive function that tends to 0 as $t \rightarrow \infty$.

Proposition. Let $u_{o}$ be a non-trivial finite energy solution of the Klein-Gordon equation. Then there is a constant $c_{o}=c_{o}$ (data) $>0$ such that

$$
\left\|u_{o}(t)\right\|_{L_{2}\left(\mathbf{R}^{n}\right)} \rightarrow c_{o}>0 .
$$

Lemma. Let $u$ be a finite energy solution of the NLKG. Then there are constants $c_{o}=c_{o}$ (data) $>0$ as above and $t_{*} \geq 1$, such that

$$
\|u(t)\|_{H_{2}^{1}(|x|>t)} \rightarrow 0 \text { as } t \rightarrow \infty
$$


and

$$
\frac{1}{8} c_{o} \leq\|u(t)\|_{L_{2}(|x| \leq t)}^{2}, t \geq t_{*} .
$$

Proof. The first statement follows from the Scattering and Energy Decay theorems. The second follows from that and the Proposition.

Proof of The Theorem. The steps of the proof of the Theorem are now obvious (following Glassey's proof for the Klein-Gordon equation [7]):

$$
\frac{1}{8} c_{o} \leq\|u(t)\|_{L_{2}(|x| \leq t)} \leq\|u(t)\|_{L_{p}(|x| \leq t)} t^{n \delta}
$$

and the results follows by taking the meanvalue over $(t, t+T)$ for $t \geq T$.

Proof of the Proposition. Let $\mathcal{F}$ denote the Fourier transform, let $B(\xi)=\left(|\xi|^{2}+\right.$ $\left.m^{2}\right)^{1 / 2}$ and $B u(x)$ be the inverse Fourier transform of $B(\xi) \mathcal{F} u(\xi)$. Define

$$
\Phi=\frac{1}{2}\left(\phi+i B^{-1} \psi\right) \text { and } \Psi=\frac{1}{2}\left(\phi-i B^{-1} \psi\right)
$$

where

$$
\phi=u(0), \psi=\partial_{t} u(0) .
$$

Then the solution $u$ of the Klein-Gordon equation can be written in the form

$$
u(t)=\exp (i t B) \Phi+\exp (-i t B) \Psi
$$

and, using duality, we have

$$
\int|u(t)|^{2} d x=\int|\Phi|^{2} d x+\int|\Psi|^{2} d x+2 \operatorname{Re} \int \exp (2 i t B) \Phi \bar{\Psi} d x .
$$

Now, by Parseval's formula, using the notation $\hat{v}=\mathcal{F} v$, we obtain

$$
\int \exp (2 i t B) \Phi \bar{\Psi} d x=\int \exp (2 i t B(\xi)) \hat{\Phi}(\xi) \overline{\hat{\Psi}}(\xi) d \xi
$$

Since $\operatorname{grad}_{\xi} B(\xi) \neq 0$ for $\xi \neq 0$, and $\Phi, \Psi$ belong to $L_{2}$, as well as their Fourier transforms (so that the products belong to $L_{1}$, respectively), we can apply the (generalized) Riemann-Lebesgue lemma to see that the right hand side tends to 0 as $t \rightarrow \infty$. Since

$$
\int|\Phi|^{2} d x+\int|\Psi|^{2} d x=\int|\phi|^{2} d x+\int\left|B^{-1} \psi\right|^{2} d x
$$

the Proposition is proved.

Appendix 1. Strichartz estimates. Let $u$ be a solution of the Klein-Gordon equation with data in $X_{e}^{1 / 2}=H_{2}^{1 / 2} \times H_{2}^{-1 / 2}$. Let $0 \leq \sigma \leq \frac{1}{2}, 2<r \leq q$ and let $\delta_{q}=\frac{1}{2}-\frac{1}{q}$ and $\delta_{r}=\frac{1}{2}-\frac{1}{r}$. Then

$$
u_{o} \in L_{r}^{\mathrm{loc}}\left(\mathbf{R} ; H_{q}^{1 / 2-\sigma}\left(\mathbf{R}^{n}\right)\right)
$$

provided

$$
\begin{gathered}
(n+2) \delta_{q} \geq 1+2 \sigma, \\
n \delta_{q}+\delta_{r} \leq 1+\sigma .
\end{gathered}
$$

We may replace $L_{r}^{\text {loc }}$ by $L_{r}$ (global in time estimate) if in addition

$$
\sigma \geq \delta_{q}-\delta_{r}(\geq 0)
$$


If $r=2$ (that is $\delta_{r}=0$ ), equality in $(\beta)$ and $(\gamma)$ is not allowed. This could be handled by using weaker spaces, e.g. Besov spaces of suitable order. For the wave equation, only $(\alpha)$ and $(\beta)$ with equality matter (and local $=$ global). In this case we use homogeneous Sobolev norms. We may also replace $L_{r}$ by $H_{r}^{s}$ if we replace $\sigma$ by $\sigma+s$.

The proof is based on some duality and restriction estimates, and then a careful use of Besov space estimates. We refer the reader to [2] and [6].

Appendix 2. Conditions on the nonlinearity of $f$. Let $f(u) \in C^{1}$ with $f(\mathbf{R}) \subseteq \mathbf{R}$ and assume that

$$
\begin{gathered}
F(u)=\int_{0}^{u} f(v) d v \geq 0 \\
\left|f^{\prime}(u)\right|<|u|^{\rho_{0}-1}, \quad|u| \leq 1 \\
\left|f^{\prime}(u)\right|<|u|^{\rho_{1}-1}, \quad|u| \geq 1
\end{gathered}
$$

where

$$
1+\frac{4}{n}<\rho_{0}, \rho_{1}<\frac{n+2}{n-2}=\rho^{*}
$$

(iii) $\quad u f(u)-2 F(u) \geq \alpha F(u)$ for some $\alpha>0$ and $F$ is not flat at 0 or $\infty$.

The last condition ensures that we avoid local concentration of energy.

\section{References}

[1] P. Brenner, On $L_{p}$-decay and scattering for nonlinear Klein-Gordon equations, Math. Scand. 51 (1982), 333-360.

[2] P. BRenner, On space-time means and everywhere defined scattering operators for nonlinear Klein-Gordon equations. Math. Z. 186 (1984), 383-391.

[3] P. Brenner, On scattering and everywhere defined scattering operators for nonlinear Klein-Gordon equations, J. Differential Equations 56 (1985), 310-344.

[4] P. Brenner, Space-time means and nonlinear Klein-Gordon equations, Report, Department of Mathematics, Chalmers University of Technology and Göteborg University, 1985-19.

[5] J. Ginibre, G. Velo, The global Cauchy problem for the nonlinear Klein-Gordon equation, Math. Z. 189 (1985), 487-505.

[6] J. Ginibre, G. Velo, Generalized Strichartz inequalities for the wave equation, J. Funct. Anal. 133 (1995), 50-68.

[7] R. T. Glassey, On the asymptotic behavior of nonlinear wave equations, Trans. Amer. Math. Soc. 182 (1973), 187-200.

[8] M. GRILlakis, Regularity and asymptotic behaviour of the wave equation with a critical nonlinearity, Ann. of Math. (2) 132 (1990), 485-509.

[9] M. Grillakis, Regularity for the wave equation with a critical nonlinearity, Comm. Pure Appl. Math. 45 (1992), 749-774.

[10] P. Kumlin, A note on maximal rate of decay for solutions to some semilinear wave equations, Math. Z. 197 (1988), 465-481. 
[11] B. Marshall, W. A. Strauss, S. Wainger, $L^{p}-L^{q}$-estimates for the Klein-Gordon equation, J. Math. Pures Appl. (9) 59 (1980), 417-440.

[12] C. S. Morawetz, Time decay for the nonlinear Klein-Gordon equations, Proc. Roy. Soc. Ser. A 306 (1968), 291-296.

[13] C. S. Morawetz, W. A. Strauss, Decay and scattering of solutions of a nonlinear relativistic wave equation, Comm. Pure Appl. Math. 25 (1972), 1-31.

[14] H. Pecher, Das Verhalten globaler Lösungen nichtlinearer Wellengleichungen für grosse Zeiten, Math. Z. 136 (1974), 67-92.

[15] I. SEgal, Space-time decay for solutions of wave equations, Adv. Math. 22 (1976), 305-311.

[16] J. Shatah, M. Struwe, Regularity results for nonlinear wave equations, Ann. of Math. (2) 138 (1993), 503-518.

[17] W. A. Strauss, Decay and asymptotics for $\square u=F(u)$, J. Funct. Anal. 2 (1968), 409-457.

[18] W. A. Strauss, Non-linear scattering theory, in: Scattering Theory in Mathematical Physics, J. A. LaVita, J.-P. Marchand (eds.), D. Riedel, Dordrecht, 1974, 53-78.

[19] R. S. Strichartz, Restriction of Fourier transforms to quadratic surfaces and decay of solutions of wave equations, Duke Math. J. 44 (1977), 705-714.

[20] R. S. Strichartz, Asymptotic behaviour of waves, J. Funct. Anal. 40 (1981), 341-357.

[21] W. von WAHL, Über die klassische Lösbarkeit des Cauchy-Problems für nichtlineare Wellengleichungen bei kleinen Anfangswerten und das asymptotische Verhalten der Lösungen, Math. Z. 114 (1970), 281-299. 